\title{
ON THE CORONAL HEATING MECHANISM BY THE RESONANT ABSORPTION OF ALFVEN WAVES
}

\author{
H.Y. ALKAHBY \\ Department of Mathematics and Computer Science \\ Adelphi University \\ Garden City, NY 11530
}

(Received December 31, 1992)

\begin{abstract}
In this paper, we will investigate the heating of the solar corona by the resonant absorption of Alfven waves in a viscous and isothermal atmosphere permeated by a horizontal magnetic field. It is shown that if the viscosity dominates the motion in a high (low) - $\beta$ plasma, it creates an absorbing and reflecting layer and the heating process is acoustic (magnetoacoustic). When the magnetic field dominates the oscillatory process it creates a non-absorbing reflecting layer. Consequently, the heating process is magnetohydrodynamic. An equation for resonance is derived. It shows that resonances may occur for many values of the frequency and of the magnetic field if the wavelength is matched with the strength of the magnetic field. At the resonance frequencies, magnetic and kinetic energies will increase to very large values which may account for the heating process. When the motion is dominated by the combined effects of the viscosity and the magnetic field, the nature of the reflecting layer and the magnitude of the reflection coefficient depend on the relative strengths of the magnetic field and the viscosity.
\end{abstract}

KEY WORDS AND PHRASES. Alfven waves, magnetohydrodynamic, resonance, acoustic. 1991 AMS SUBJECT CLASSIFICATION CODES. 76N, 76Q.

\section{INTRODUCTION.}

It is well known that the solar corona is extremely hot, typical temperatures are $10^{6} K$, compared with $5 \times 10^{3} K$ in the photosphere. Consequently, thermal energy must be continually supplied to maintain this temperature against radiative cooling. Early theories of coronal heating were essentially based on the dissipation of acoustic waves or shock waves. Recent theories invoke magnetic energy dissipation as the source of thermal energy. Thus two questions must be answered: how is magnetic energy supplied to the corona, and how is it dissipated? To answer these questions, many models and dissipative mechanisms are suggested (see Priest [10], chaps 4, 5, 6; Yanowitch [12], [13]; Campos [4], [5]; Roberts [11]; Alkahby and Yanowitch [2], [3]).

Resonance absorption was suggested as a mechanism for the heating of fusion plasmas nearly 30 years go. Ionson ([8], [9]) and Hollweg [7] both concluded that resonance absorption could explain the observed heating in the solar corona. Davila [6] calculated the heating rate at the resonance layer to determine the energy dissipation in the plasma.

The aim of this paper is to investigate the heating of the solar corona by resonance absorption of Alfven waves and to calculate the kinetic and magnetic energies of an upward 
propagating magnetoacoustic waves in a viscous and isothermal atmosphere permeated by a horizontal magnetic field. Its shown that if the viscosity dominates the oscillatory process for low and high $\beta$ plasma $\left(\beta=\frac{\text { gas pressure }}{\text { magnetic pressure }}\right)$, it creates an absorbing and reflecting transition region, in which the reflection and the waves modification take place. Below it the motion is adiabatic and the effects of the viscosity and the magnetic field are negligible. Above it the motion will be influenced by the combined effects of the viscosity and the magnetic field. Consequently, the heating mechanism is acoustic for large $\beta$ and magnetoacoustic for small $\beta$.

When the magnetic field dominates the motion, it generates a non-absorbing reflecting layer. This result is expected because of the dissipationless nature of the magnetic field. As a result of that, the heating mechanism is magnetohydrodynamic and resonance will occur for many values of the magnetic field and of the frequency. At the resonance frequency, the magnetic and the kinetic energies increase to very large values which may account for the heating process.

Finally, if neither the viscosity nor the magnetic field dominates the motion, the nature of the transition region and the magnitude of the reflection coefficient depend on the relative strengths of the viscosity and the magnetic field.

\section{FORMULATION OF THE PROBLEM.}

We will consider an isothermal atmosphere, which is viscous and thermally nonconducting, occupies the upper half-space $z>0$. It will be assumed that the gas is under the influence of a uniform horizontal magnetic field and that is has infinite electrical conductivity. We will investigate small oscillations about equilibrium which depend only on the time $t$ and on the vertical coordinate $z$. Let $p, \rho, w$, and $B$ be the perturbations in the pressure, density, vertical velocity, and the magnetic field strength, and $P_{0}, \rho_{0}, T_{0}$, and $B_{0}$ are the equilibrium quantities. The equilibrium pressure and density,

$$
P_{0}(z) / P_{0}(0)=\rho_{0}(z) / \rho_{0}(0)=\exp (-z / H),
$$

are determined by the gas law, $p_{0}=R T_{0} \rho_{0}$ and the hydrostatic equation, $p^{\prime}+g \rho_{0}=0$, where $R$ is the gas constant, $g$ is the gravitational acceleration, and $H=R T_{0} / g$ is the density scale height. The linearized equations of motion are:

$$
\begin{gathered}
\rho_{0} w_{t}+p_{z}+g \rho+\left(B_{0} / 4 \pi\right) B_{z}=4 \mu w_{z z} / 3 \\
\rho_{t}+\left(\rho_{0} w\right)_{z}=0, \\
B_{t}+B_{0} w_{z}=0, \\
p_{t}-g \rho_{0} w+c^{2} \rho_{0} w_{z}=0 .
\end{gathered}
$$

These are, respectively, the equation for the change in the vertical momentum, the mass conservation equation, the equation for the rate of change of the $x$-component of the magnetic field, and the pressure equation which is obtained from the adiabatic equation and the continuity equation and $c=\sqrt{\gamma R T_{0}}=\sqrt{\gamma g H}=\sqrt{\gamma p_{0} / \rho_{0}}$ is the adiabatic sound speed. Here $\mu$ is the dynamic viscosity coefficient, which is assumed to be constant, and the subscripts $z$ and $t$ denote differentiations with respect to $z$ and $t$ respectively. We will consider solutions which are harmonic in time, i.e., $w(z, t)=W(z) \exp (-i \omega t)$.

It is more convenient to rewrite the equations in dimensionless form; $z^{\prime}=z / H, \omega_{a}=c / 2 H$, $W^{\prime}=w / c, \omega^{\prime}=\omega / \omega_{a}, t^{\prime}=t \omega_{a}, \alpha=c_{A}^{2} / c^{2}, \mu^{\prime}=2 \mu /\left(3 \rho_{0}(0) c H\right), \eta=\alpha-i \omega^{\prime} \mu^{\prime}, c_{A}=B_{0} / \sqrt{4 \pi \rho_{0}(0)}$ is the Alfven speed at $z=0$, and $\omega_{a}$ is the adiabatic cutoff frequency. The primes can be omitted, since all 
variables will be written in dimensionless form from now on. One can eliminate $P, \rho$ and $B$ to have an equation for $W(z)$ only, by applying $\frac{\partial}{\partial t}$ to (2.2) and substituting (2.3) - (2.5),

$$
\left(D^{2}-D+\omega^{2} / 4\right) W(z)+\eta e^{z} D^{2} W(z)=0,
$$

where $D=d / d z$.

Boundary Condztions: To complete the formulation of the problem, certain conditions must be imposed to ensure a unique solution. Physically relevant solutions must satisfy the dissipation condition (DC), which requires the finiteness of the rate of the energy dissipation in an infinite column of fluid of unit cross-section. Since the dissipation function depends on the squares of the velocity gradients, this implies

$$
\int_{0}^{\infty}\left|W_{z}\right|^{2} d z<\infty
$$

A boundary condition is also, required at $z=0$ and we shall set

$$
W(0)=1,
$$

by suitably normalizing $W(z)$. It will be seen that the boundary conditions (2.7) and (2.8) will determine a unique solution to within a multiplicative constant.

\section{REDUCTION TO THE HYPERGEOMETRIC EQUATION AND SOLUTIONS.}

The differential equation (2.6) can be reduced to the hypergeometric equation by introducing a new dimensionless variable,

$$
\xi=-\exp (-z) / \eta
$$

then equation (2.6) will be transformed into

$$
\left[\xi(1-\xi) D^{2}+(1-2 \xi) D-\omega^{2} / 4\right] W(\xi)=0,
$$

where $D=d / d \xi$ and $\arg (-\xi)=-\arg (\eta)$. Equation (3.2) is a special case of the hypergeometric equation

$$
\left[\xi(1-\xi) D^{2}+(c-(a+b+1) \xi) D-a b\right] W(\xi)=0,
$$

with $c=1, a=\frac{1}{2}+s$ and $b=\frac{1}{2}-s$, where $s=\sqrt{1-\omega^{2}} / 2$ for $\omega<1, \quad s=0$ for $\omega=1$ and $s=i \sqrt{\omega^{2}-1} / 2=i k$ for $\omega>1, k$ is the adiabatic wave number and we will be interested in the last case.

For fixed value of $|\eta|>0$, the point $\xi=0$ corresponds to $z=\infty$, the point $\xi_{0}=-1 / \eta=\exp (-\log |\eta|+i(\pi-\theta))$ where $\theta=\arg (\eta)$ to $z=0$, and the segment connecting these points in the complex $\xi$ - plane to $z>0$.

For $|\xi|<1$, equation (3.2) has two linearly independent solutions of the form

$$
\begin{gathered}
W_{1}(\xi)=F(a, b, c, \xi), \\
W_{2}(\xi)=W_{1}(\xi) \ln \xi+\sum_{n=1}^{\infty} \frac{(a)_{n}(b)_{n}}{(n !)^{2}} \xi^{n}[\psi(a+n)-\psi(a)+\psi(b+n)-\psi(a)-2 \psi(n+1)+\psi(n)]
\end{gathered}
$$

where $a=\frac{1}{2}+s, b=\frac{1}{2}-s$ and $F$ is the hypergeometric function. For $|\xi|>1$ it is convenient to choose

$$
\begin{gathered}
W_{a}(\xi)=\xi^{-a} F\left(a, a, 2 a, \xi^{-1}\right), \\
W_{b}(\xi)=\xi^{-b} F\left(b, b, 2 b, \xi^{-1}\right) .
\end{gathered}
$$


The second solution $W_{2}(\xi)$ will be ruled out by the dissipation condition. Finally, the solution of equation (3.2) is

$$
W(\xi)=C_{a} W_{1}(\xi)=C_{a} F(a, b, c, \xi),
$$

where $C_{a}$ is a constant which can be determined by the boundary condition $W(0)=1$ at $\xi_{0}=\exp (-\log |\eta|+i(\pi-\theta))$. For $|\xi|>1$ and $\omega \neq 1$ the analytic continuation of $W(\xi)$ is

$$
W(\xi)=C_{a}\left[\frac{\Gamma(b-a)}{\left(\Gamma^{2}(b)\right.}(-\xi)^{-a} F\left(a, a, 2 a, \xi^{-1}\right)+\frac{\Gamma(a-b)}{\Gamma^{2}(a)}(-\xi)^{-b} F\left(b, b, 2 b, \xi^{-1}\right)\right]
$$

When $\omega=1$ and $|\xi|>1$, the analytic continuation is given by

$$
W(\xi)=C_{a} \frac{1}{\hbar}(-\xi)-\frac{1}{2} \Sigma_{0}^{\infty} \frac{\Gamma\left(n+\frac{1}{2}\right)^{2}}{\Gamma\left(\frac{1}{2}\right)^{2} \Gamma(n+1)^{2}}\left(\xi^{-n}\right)\left[\ln (-\xi)+2 \psi(n+1)-\psi\left(n+\frac{1}{2}\right)-\psi\left(\frac{1}{2}-n\right)\right] .
$$

\section{ASYMPTOTIC ESTIMATE FOR THE KINETIC AND MAGNETIC ENERGIES.}

For a bounded $z$ - interval $\xi^{-1}=O(\eta)$, using equation (3.1) and retaining the most significant terms in equation (3.9), we have asymptotically as $\eta \rightarrow 0$

$$
W(z) \sim C a\left[\frac{\Gamma(b-a)}{\Gamma^{2}(b)} \exp \left[\left(\frac{1}{2}+i k\right)(z+\ln (\eta))\right]+\frac{\Gamma(a-b)}{\Gamma^{2}(a)} \exp \left[\left(\frac{1}{2}-i k\right)(z+\ln (\eta))\right]\right]
$$

Úsing the boundary condition $W(0)=1$ equation (4.1) will be written like

$$
W(z) \sim\left[\frac{1}{(1+R C)}\left[\exp \left(\frac{1}{2}+i k\right) z+R C \exp \left(\frac{1}{2}-i k\right) z\right]\right]
$$

where the reflection coefficient $R C$ is defined by

$$
\begin{gathered}
R C=\exp \left(2 k \theta+i \theta_{1}\right), \\
\theta_{1}=\arg (R C)=\theta_{2}=2 k \ln |\eta|, \\
\theta_{2}=2 \arg \Gamma(2 i k)-4 \arg \Gamma\left(\frac{1}{2}+i k\right) .
\end{gathered}
$$

It is clear that $|R C|=\exp (2 k \theta),|R C|=1$ if $\theta=0$ and $R C=|R C| \exp \left(i \theta_{1}\right)$.

The time average of the kinetic energy $\left(K_{E}\right)$ can be evaluated from equation (4.2) and one obtains

$$
K_{E}=\rho|W|^{2}=\frac{1+|R C|^{2}+2|R C| \cos \left(2 k z-\theta_{1}\right)}{2|1+R C|^{2}} .
$$

It follows from equation (2.4) that the magnetic energy $\left(M_{E}\right)$

$$
M_{E}=\frac{B_{0}^{2}}{8 \pi}\left|W_{z}\right|^{2} \propto\left(K_{E}\right)
$$

From equation (4.6) and (4.7) we have the following observations

(I) When the viscosity dominates the oscillatory motion $(\alpha \ll \mu)$, and for large or small $\beta=\frac{2 c^{2}}{r c_{A}^{2}}$, $\theta \rightarrow-\frac{\pi}{2},|R C|=\exp (-\pi k)$, and the maximum and the minimum values of the kinetic energy are

and attained when

$$
\operatorname{Max}\left(K_{E}\right)=\frac{|R C|^{2}+2|R C|+1}{2|R C+1|}=\frac{\cosh \pi k+1}{2\left(\cosh \pi k+\cos \theta_{1}\right)}
$$

$$
\begin{gathered}
{ }^{z}{ }_{M}=\frac{\theta_{1} \pm 2 n \pi}{2 k} \\
\min \left(K_{E}\right)=\frac{|R C|^{2}-2|R C|+1}{2|R C+1|}-\frac{\cosh \pi k-1}{2\left(\cosh \pi k+\cos \theta_{1}\right)}
\end{gathered}
$$


and attained when

$$
z_{m}=\frac{\theta_{1} \pm(2 n+1) \pi}{2 k}
$$

The magnitude of the reflection coefficient can be obtained from the maximum and the minimum values of the kinetic energy. Let $d=\sqrt{\frac{\max \left(K_{E}\right)}{\min \left(K_{E}\right)}}$, then

$$
|R C|=\frac{d-1}{d+1}<1
$$

If $\theta_{1} \rightarrow \pm(\pi+2 n \pi)$ the magnitude of the reflection coefficient will be unchanged.

(II) When the magnetic field dominates the oscillatory process $(\mu \ll \alpha)$ and for small $\beta, \theta \rightarrow 0,|R C| \rightarrow 1, \min \left(K_{E}\right) \rightarrow 0$, and

$$
\operatorname{Max}\left(M_{E}\right) \rightarrow \operatorname{Max}\left(K_{E}\right) \rightarrow \frac{1}{1+\cos \theta_{1}}
$$

If $\theta_{1} \rightarrow \pm(\pi+2 n \pi)$ then

$$
\operatorname{Max}\left(M_{E}\right) \rightarrow \operatorname{Max}\left(K_{E}\right) \rightarrow \infty
$$

Consequently, the magnetic and kinetic energies will be increased to very large values when

$$
\ln |\eta|=\frac{\theta_{2} \pm(\pi+2 n \pi)}{2 k}=\frac{\theta_{2} \pm(\pi+2 n \pi)}{4 \pi} \lambda
$$

where $\lambda$ is the wavelength. We call this equation "the resonance equation". The resonance equation states that resonance will occur for many values of the frequency or of the magnetic field if the wavelength is matched with the strength of the magnetic field.

\section{DISCUSSIONS AND CONCLUSIONS.}

It is known that an initiated disturbance results in a sound wave which propagates radially away from the source with speed $c$. In the presence of a magnetic field, variations in the atmospheric pressure will causes disturbances of the magnetic field -lines. Thus any attempt to initiate a sound wave will result in the variation in the magnetic field. As a conclusion, the sound will not propagate with sound speed $c$, and the directionality of the magnetic field will render wave propagation anistropic. As a result of this, the wave speed $W_{S}$ will be $S_{S} \leq W_{S}<F_{S}$, where $S_{S}$ is the slow speed, $F_{S}$ is the fast speed and they are defined by

$$
F_{S}=\sqrt{c^{2}+c_{A}^{2}}, S_{S}=\sqrt{\frac{c^{2} c_{A}^{2}}{c^{2}+c_{A}^{2}}} .
$$

If follows from our observations in section 4 , that for large $\beta, S_{S} \leq W_{S}<F_{S}$, and consequently the heating process is either acoustic or magnetoacoustic. For small $\beta$ we obtain $S_{S}<W_{S} \leq c_{A}$ and in conclusion the heating mechanism is magnetohydrodynamic. At the resonance frequency the magnetic and the kinetic energies will increase to very large value and that may account for the heating process.

ACKNOWLEDGEMENT. I would like to express my sincere thanks to Professor Michael Yanowitch for his continuing encouragement, support, and invaluable criticism during the preparation of this work.

\section{REFERENCES}

1. ABRAMOWITZ, M. \& STEGUN, I., Handbook of Mathematical Functions, National Bureau of Standards, Washington, D.C., 1964.

2. ALKAHBY, H.Y. \& YANOWITCH, M., The effects of Newtonian cooling on the reflection of vertically propagating acoustic waves in an isothermal atmosphere, Wave Motion 11 (1989), 419-426. 
3. ALKAHBY, H.Y. \& YANOWITCH, M., Reflection of vertically propagating waves in a thermally conducting isothermal atmosphere with a horizontal magnetic field, Geophys. Astroph. Fluid Dynam. 56 (1991), 227-235.

4. CAMPOS, L.M.B.C., On magnetoacoustic-gravity waves propagating or standing vertically in an atmosphere, J. Phys. A. 16 (1983a), 417-437.

5. CAMPOS, L.M.B.C., On viscous and resistive dissipation of hydrodynamic and hydromagnetic waves in atmospheres, J. Mec. Theor. Appl. 2 (1983b), 861-891.

6. DAVILA, J.M., Heating of the solar corona by the resonant absorption of Alfven waves, $A p$. J. 317 (1987), 514-521.

7. HOLLWEG, J.V., Resonances of coronal loops, Ap. J. 277 (1984), 392-403.

8. IONSON, J.A., Resonant electrodynamic heating of steller loops; An LRC circuit analog, Ap. J. 271 (1982), 318-334.

9. IONSON, J.A., Electrodynamics coupling in magnetically confined $x$-ray plasmas of astrophysical origin, Ap. J. 271 (1983), 778-792.

10. PRIEST, E.R., Solar Magnetohydrodynamics, D. Reidel Pub. Co., 1984.

11. ROBERTS, B.H., Waves in the solar atmosphere, Geophys. Astroph. Fluid Dynam. 62 (1991), 83-100.

12. YANOWITCH, M., The effect of viscosity on vertical oscillations of an isothermal atmosphere, Can. J. Phys. 45 (1967), 2003-2008.

13. YANOWITCH, M., Vertically propagating hydromagnetic waves in an isothermal atmosphere with a horizontal magnetic field, Wave Motion 1 (1979), 123-125. 


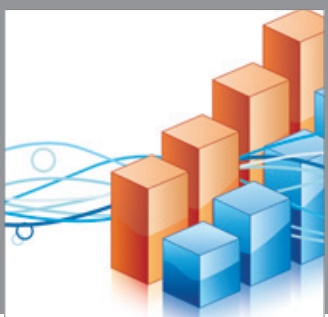

Advances in

Operations Research

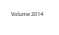

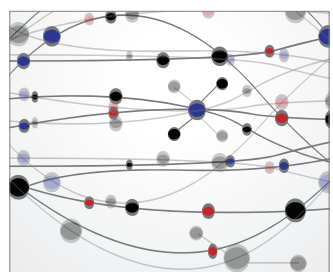

\section{The Scientific} World Journal
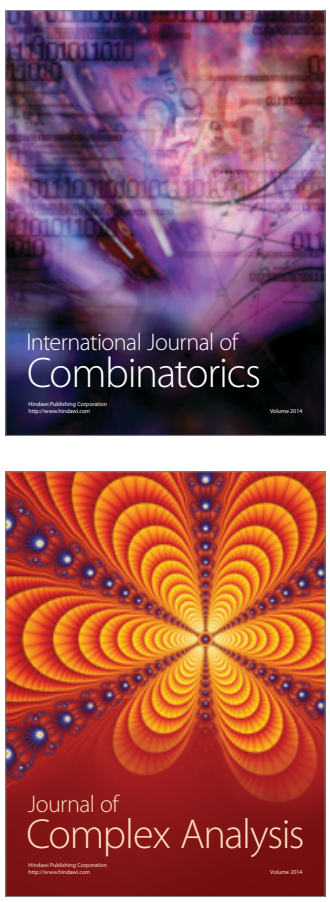

International Journal of

Mathematics and

Mathematical

Sciences
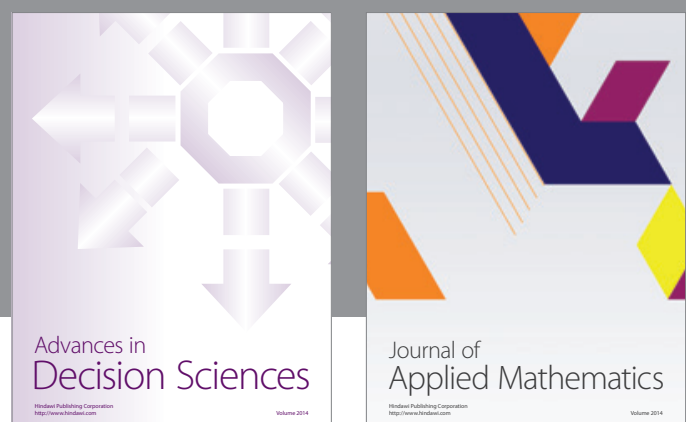

Journal of

Applied Mathematics
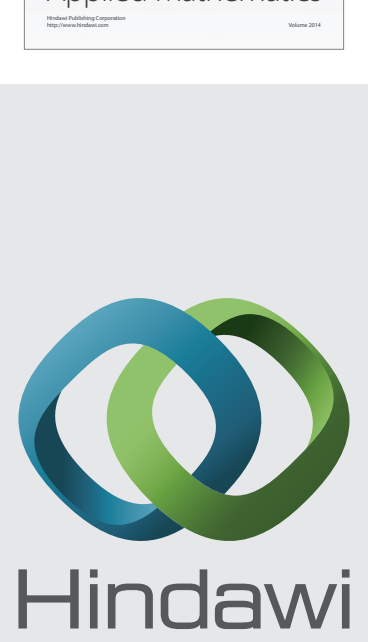

Submit your manuscripts at http://www.hindawi.com
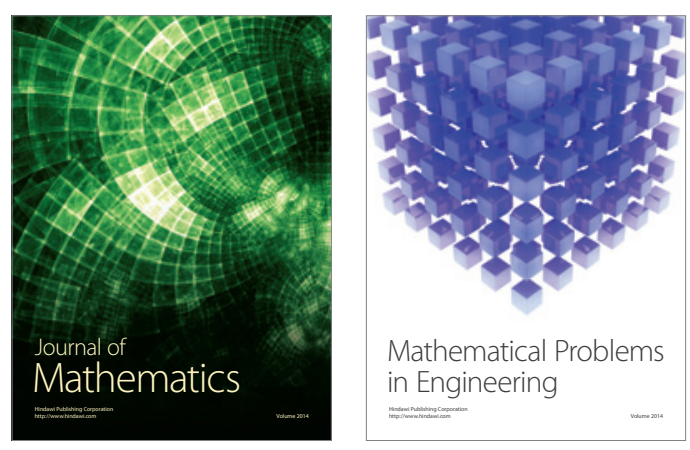

Mathematical Problems in Engineering
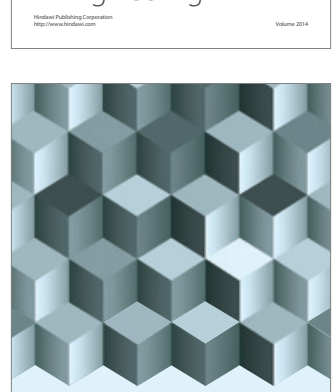

Journal of

Function Spaces
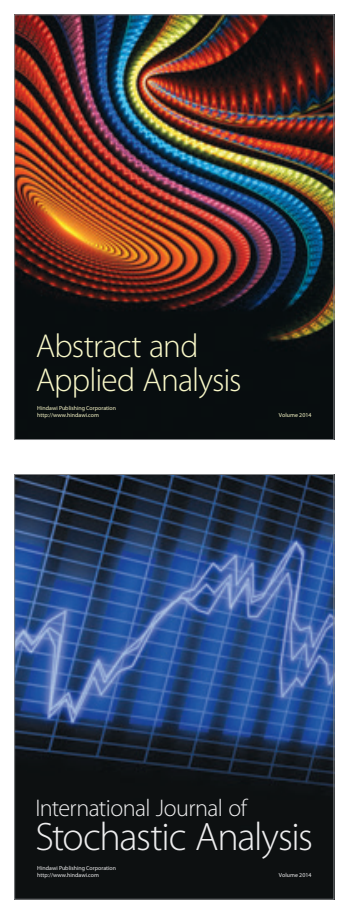

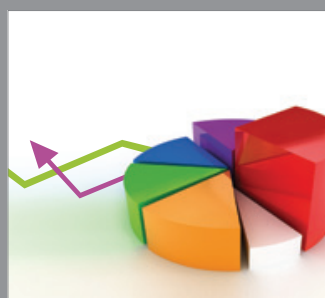

ournal of

Probability and Statistics

Promensencen
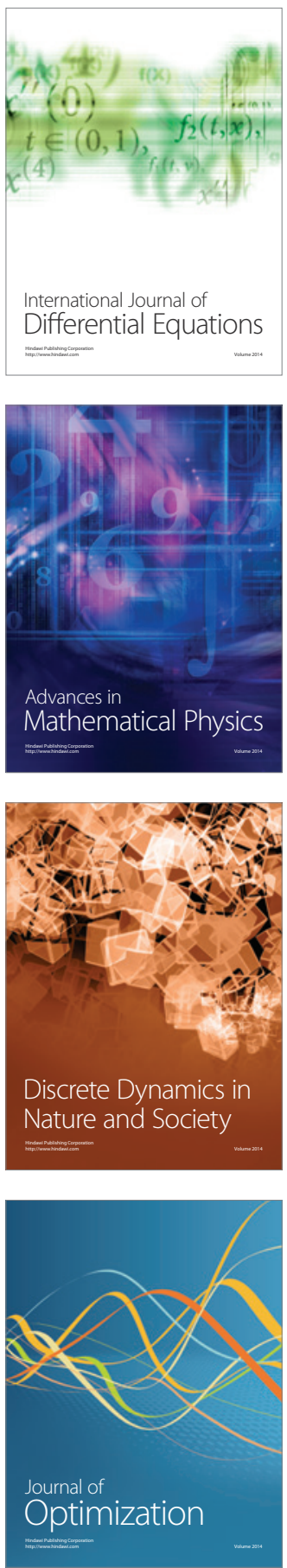